\title{
Diagnostics of liquid properties by the example of bioorganic solutions
}

\author{
Alexander Malov, Anna Neupokoeva , Alexandra Sambyalova \\ Department of Medical and Biological Physics, Irkutsk State Medical University, Irkutsk, Krasnogo Vosstaniya Str. 1, \\ 664009, Russia \\ *e-mail: annett 2005@inbox.ru
}

\begin{abstract}
Two methods of monitoring the structure modifications of protein solutions are considered: using the speckle patterns in real time and using crystallograms. It is shown that the proposed methods of image processing correlate with each other and allow the tracing of the qualitative structure modifications. (C) 2016 Journal of Biomedical Photonics \& Engineering.
\end{abstract}

Keywords: speckle, crystallogram, image processing, contour, structure monitoring, autocorrelation.

Paper \#2889 received 2016.01.08; revised manuscript received 2016.03.02; accepted for publication 2016.03.09; published online 2016.03.30. doi: 10.18287/JBPE16.02.010304

\section{References}

1. A. N. Malov, and A. V. Neupokoyeva, "Chess board method for the analysis of speckle images," Proceedings of the 10-th Int. Conf. HoloExpo-2013, 257-264 (2013) [in Russian].

2. I. E. Golub, A. N. Malov, A. V. Neupokoyeva, and L. V. Sorokina, "Registration of laser-induced microstructure changes of blood plasma by the wavelet analysis of speckle patterns," Proceedings of V Troitsk Conference Medical Physics and Innovations in Medicine 1, 252-254 (2012) [in Russian].

3. M. A. Vilenskii, D. N. Agafonov, D. A. Zimnyakov, V. V. Tuchin, and R. A. Zdrazhevskii, "Specklecorrelation analysis of the microcapillary blood circulation in nail bed," Quantum Electron. 41(4), 324-328 (2011).

4. I. V. Fedosov, and V. V. Tuchin, "The space-time correlation of the intensity of a speckle field formed as a result of scattering of focused coherent radiation by a capillary liquid flow containing scattering particles," Opt. Spectrosc. 93(3), 434-438 (2002).

5. A. N. Malov, E. S. Musatova, A. U. Setejkin, and S. V. Zinoviev "Laser nanoclasterization processes simulation," Proceedings of the 8th Russia and China Pharmaceutical Forum Modern Problems of Nanopharmacology, 66 - 67 (2011).

6. V. N. Shabalin, and S. N. Shatokhina, Morphologies of Human Biological Liquids, Khrizostom, Moscow (2001) [in Russian].

7. A. N. Malov, E. S. Musatova, and A. Y. Seteikin, "Crystallographic registration of the results of laser nanocrystallisation of bioorganic liquids," Proceedings of V Troitsk Conference Medical Physics and Innovations in Medicine 2, 80-82 (2012) [in Russian].

8. R. Paringer, A. Kupriyanov, and N. Ilyasova, "Dendritic crystallogram images classification," JBPE 1(2), 135138 (2015).

9. N. Y. Ilyasova, A. V. Kupriyanov, and A. G. Khramov, Information Technologies of Image Analysis in the Problems of Medical Diagnostics, Radio i svyaz, Moscow, (2012) [in Russian].

10. Y. Y. Tarasevich, "Mechanisms and models of the dehydration self-organization in biological fluids," Phys.Usp. 47(7), 717-728 (2004).

11. A. N. Malov, A. Y. Seteikin, A. V. Neupokoeva, E. S. Musatova, I. E. Golub, L. V. Sorokina, and A. A. Vaichas, "The laser radiation action on the biological objects," Optik - International Journal for Light and Electron Optics 124(23), 6034-6041 (2013).

12. J. S. Bendat, and A. G. Piersol, Engineering Applications of Correlation and Spectral Analysis, John Wiley\&Sons, New York (1980). ISBN: 978-0471570554. 


\section{Introduction}

In the monitoring of laser impact on multicomponent or hardly standardisable media (protein solutions, blood plasma, petrol, gelatine-based holographic media) the possibility of fast obtaining the processing results could significantly increase the efficiency of the impact, or could allow the choice of the impact parameters corresponding to the specific features of the particular medium [1-4]. For such media, represented by the solutions of complex organic molecules, a few methods were proposed to determine the structure inhomogeneities.

One of the simplest and clear methods for monitoring the structure modifications in liquid media is crystallography, in which the segment size of a crystallogram is directly related to the characteristic size of inhomogeneities in biological liquids [5-9].

The phenomenon of dehydration self-organisation is chosen to be the basis of one of the medical diagnostic methods, the method of wedge-shaped dehydration [810] that consists in the following. A droplet of the studied biological liquid (for the control samples and the ones exposed to laser radiation) with the volume 0.01 $\mathrm{ml}$ is deposited on the defatted object glass and dried tilted at the angle $25-30^{\circ}$ and dust-protected at room temperature $20-25^{\circ} \mathrm{C}$ and relative air humidity $65-70 \%$ during 18-24 hours. Using this method the crystallograms of the preparations of the studied biological liquids were obtained. The drawback of this method is the impossibility of controlling the liquid structure in real time, since the preparation (drying) of a crystallogram takes a few hours.

In Ref. [7] it was shown that the size of the crystals essentially changes under the exposure of the glycine solution with the concentration $5 \%$ to the radiation of a red helium-neon laser with the wavelength $633 \mathrm{~nm}$ during $15 \mathrm{~min}$, the radiation power density being nearly $0.6 \mathrm{~mW} / \mathrm{cm}^{2}$ (Fig. 1). Thus, when the crystallograms are formed in dry films from $5 \%$ solution of glycine, their structure is determined by the nucleation centres, which, in turn, depend upon the state of the initial solution. The bioorganic molecules in a solution have the aqueous environment and form clusters with different size and configuration. When the solution is dried, the large-size clusters having smaller velocity of chaotic motion are first of all fixed at the substrate and play the role of nucleation centres for the growth of crystals in the film.

Then the epitaxial growth of the film occurs at the expense of the later crystallised low-molecular fractions fixed on the dendrite-like base. In this way a ray-shaped pattern of lamellas with epitaxial recrystallization on the ray part playing the role of the substrate for the exsiccant solution is formed. The reduction of the thickness of the lamella rays after the laser irradiation shows that in the irradiated solution the cluster size is considerably reduced, which leads to the size reduction of the nucleation centres in the course of the film drying $[5,7,11]$.

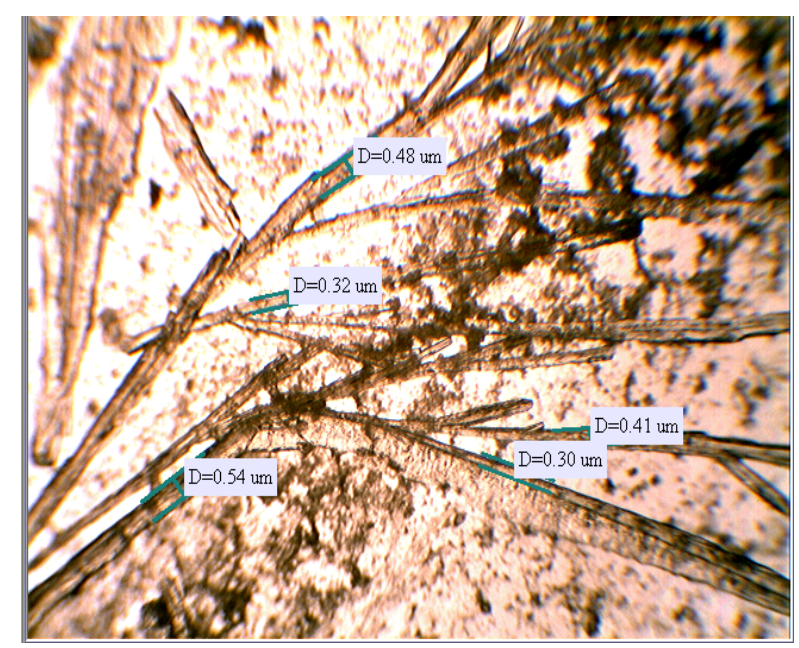

(a)

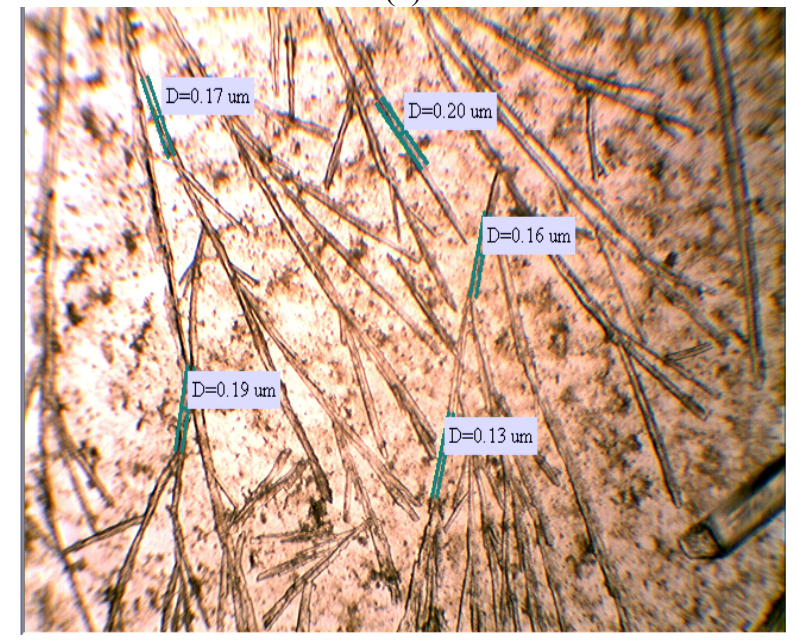

(b)

Fig. $15 \%$ glycine solution under the $50 \times$ magnification. (a) the control sample; (b) after laser treatment system.

An alternative method of structure monitoring is to use the speckle pattern of the studied liquid. Since the size of the organic molecular associates, e.g., of those having the protein nature, can by many times exceed the size of an individual molecule, such complexes will manifest themselves as essential fluctuations of the refractive index in the solution and will be the objects at which the diffraction of the laser radiation occurs. Speckle patterns are formed as a result of interaction of coherent laser radiation with nonuniformity medium. According to the diffraction laws, the characteristic spot size of the speckle pattern is inversely proportional to the inhomogeneity size. At present the speckle optical methods of diagnostics of liquids, biological objects, and solid surfaces are intensely developed, since the speckle patterns contain a large amount of information about the size, concentration, and motion velocities of in-homogeneities within the bulk sample.

On the other hand, there is an urgent issue of selecting the useful information contained in speckle patterns from the random components related to the presence of multiple optical surfaces, parasitic illumination, etc., in the optical schemes. Thus, the aim 
of the present paper is to elaborate an algorithm for processing speckle patterns and crystallograms, as well as to introduce numerical criteria that would allow the efficient comparison of images, obtained at different stages of monitoring.

\section{Materials and Methods}

In the present work the object of study including the formation of crystallograms was the Grippferon solution, the main component of which is interferon alpha-2b, the protein-nature factor that provides antivirus immunity. The Grippferon solution in a cuvette was subjected to the laser radiation (the mean wavelength $655 \mathrm{~nm}$, the intensity about $80 \mathrm{~mW} / \mathrm{cm}^{2}$ ) during 20 minutes. Then the irradiated and the control solution was poured on similar glass substrates and kept at room temperature for obtaining the crystallograms. The series consisted of ten similar experiments, in which similar-type crystallograms were obtained for the appropriate time of exposure.

The speckle patterns were obtained from the gelatine solution in the gelling process of its. For this goal the spatial distribution of laser radiation after passing through the cuvette with gelatine solution was recorded by means of a digital photo camera. The gelatine solution with the concentration $10 \%$ (by the mass of dry gelatine) kept in the water bath at the temperature $50^{\circ} \mathrm{C}$, was placed in a cuvette near the focus of the laser beam (Fig. 2). The scattered radiation was recorded immediately after the installation of the solution into the setup (the beginning of gelling) and then each 5 minutes until the final time of 30 minutes, during which the solution turned into gel.

The wavelength of the probe radiation was $655 \mathrm{~nm}$, the intensity was about $150 \mathrm{~mW} / \mathrm{cm}^{2}$. The preparation of the gelatine solution, the process of gelling, and the recording of speckle patterns was repeated 5 times under the similar conditions. The dynamics of the numerical parameters of the speckle patterns was similar in all experiments.

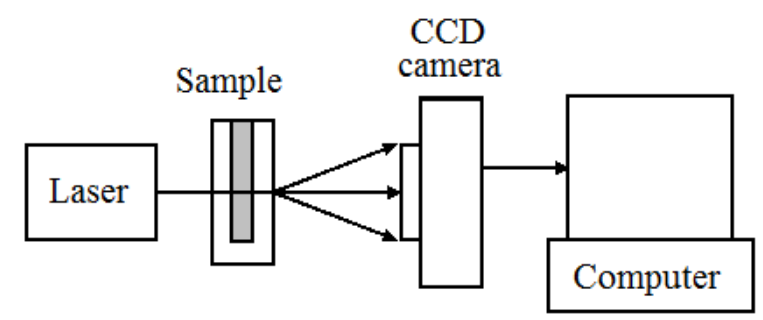

Fig. 2 Schematic diagram of the experimental setup.

The resulting speckle patterns were used to calculate the autocorrelation coefficient. The correlation is known to be expressed by the convolution integral, where the functions $g(x)$ and $h(x)$ correspond to the intensity distribution functions in the studied patterns:

$$
K(x, y)=\iint g(\xi, \eta) \cdot h(x-\xi, y-\eta) d \xi d \eta=F^{-1}\{G(\xi, \eta) \cdot H(\xi, \eta)\}
$$

For the autocorrelation function we have

$$
A(x, y)=\iint g(\xi, \eta) \cdot g(x-\xi, y-\eta) d \xi d \eta=F^{-1}\{G(\xi, \eta) \cdot G(\xi, \eta)\}
$$

For the calculations we used the convolution theorem, i.e., at first the Fourier transform was calculated for each pattern using the FFT algorithm, then the Fourier transforms were multi-plied, and then the inverse Fourier transform was calculated. The resulting autocorrelation function is a function of two coordinates $A(x, y)$. Numerically this is a matrix, the dimension of which depends on the initial size of the speckle pattern. The graphic representation of this matrix looks like a pyramid, the surface of which depends upon the properties of the studied pattern (Fig. 3 ). For convenience we took square speckle patterns, and therefore, the coefficients of autocorrelation were represented by square matrices.

In the vicinity of zero a certain peak is observed, the width of which can be estimated at some level, e.g., 0.9 or $1 / \mathrm{e}$ of the maximum. The advantage of this method is that the data of the entire speckle pattern are taken into account, and the drawback is the dependence of the autocorrelation coefficient upon the brightness of the speckle pattern, which can make the interpretation difficult, as well as the loss of data on the structure of the autocorrelation pyramid, if only the central peak width is used as a numerical parameter.

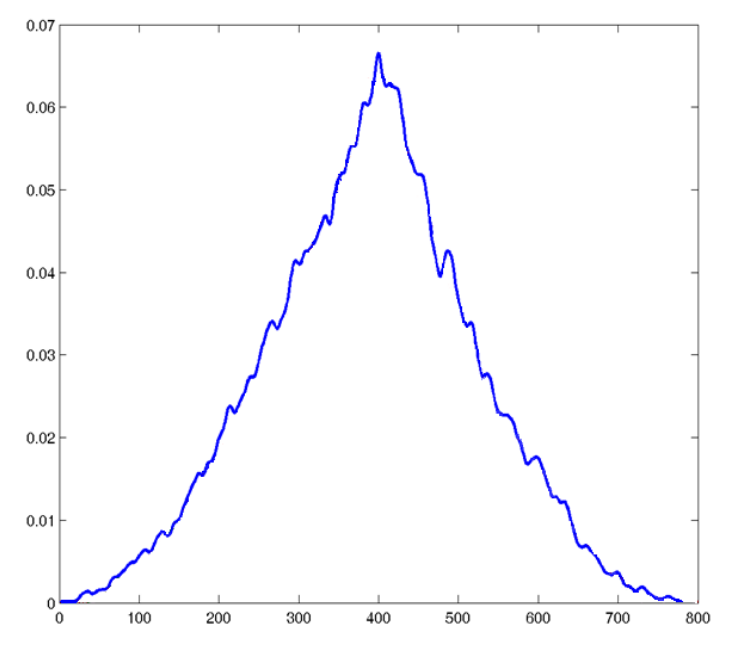

Fig. 3 Typical view of the autocorrelation function in the diagonal section.

The real autocorrelation function (Fig. 3) has complex shape that makes the determination of its halfwidth difficult. The local oscillations of the function are due to the fact that the speckle pattern at finite sampling can be considered as a quasiperiodic function, which gives rise to the oscillations. To estimate the correlation radius, one can take the separation between the peak 
centre and the nearest local maximum (this distance corresponds to the mean size of a speckle). The error of such method can be estimated as $7-10 \%$ [12].

As an alternative method of speckle pattern processing we used the algorithm based on the binarisation of the obtained image. This method was also used to derive numerical estimates from the crystallograms.

The image processing program was written basing on the library OpenCV (Open Source Computer Vision Library), a computer vision library with open source code. In medicine OpenCV is used in the processing of $\mathrm{X}$-ray/MRT/CT images. For the processing of crystallograms in the official documentation of the OpenCV library, we used the algorithm of contour processing. The procedure includes the image binarisation according to the chosen level. All pixels with the brightness above this level are considered white, and all other pixels are considered black. Then the mean width and height of the obtained spots and their mean area are calculated. The resulting data (images with contour boundaries, numerical characteristics) are written in a file and displayed on the screen. We also added the possibility of filtering small contours (in the final image they are shown in a different colour and are not taken into account in calculations) [11].

\section{Experimental results}

The typical crystallogram view and the results of processing are presented in Fig. 4. The pattern binarisation level was chosen similar in all cases to provide the comparability of the patterns in the series

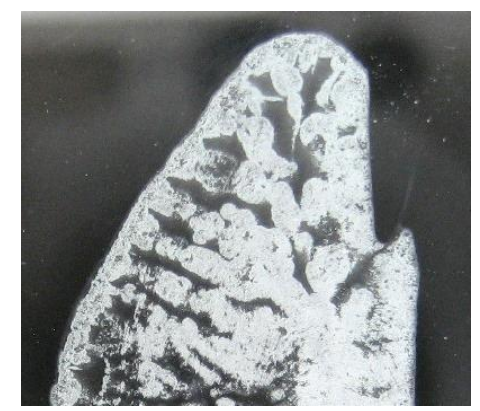

(a)

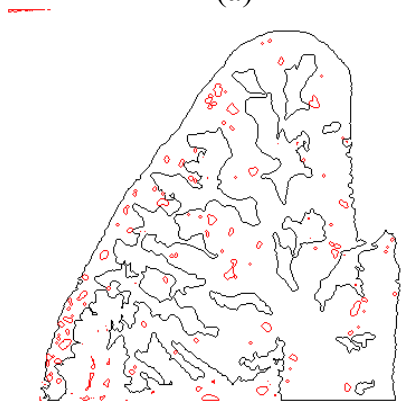

(c) and amounted to $130 \%$ of the mean brightness of the pattern, calculated in the program. Lighter contours are those not taken into account in the calculations. The threshold contour size below which a contour was excluded from the general statistics was chosen $10 \%$ of the current mean size. For example, if the program returned the mean contour size of 40 pixels, then the contours smaller than 4 pixels were neglected. The results of the calculations are presented in Fig. 5. Before the laser impact the mean speckle size was $X=57.6$ pixels by $\mathrm{Y}=61.2$ pixels. After the impact the mean pixel size appeared to be $\mathrm{X}=38.6$ pixels by $\mathrm{Y}=45.3$ pixels. One pixel of the image corresponds to the inhomogeneity of about $30 \mu \mathrm{m}$.

The program calculating the mean contour size was also used to analyse the speckle pat-terns (Fig. 6). The results of the processing of the speckle patterns recorded at different moments of time starting from the beginning of the gelling process are presented in Fig. 7.

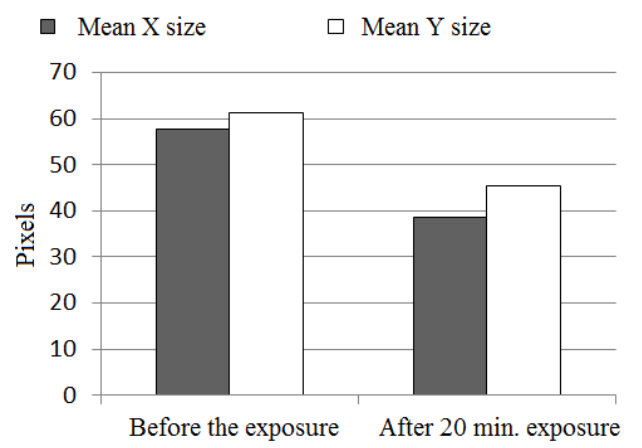

Fig. 5 Change of the characteristic pattern scale, obtained using the computer processing.

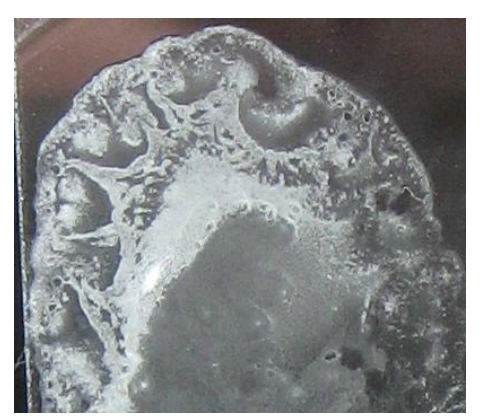

(b)

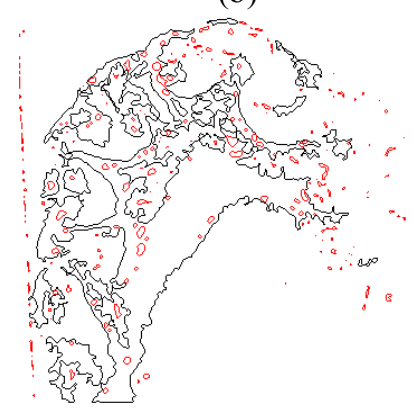

(d)

Fig. 4 View of the solution crystallogram without the laser radiation impact (a) and after the 20 minutes of exposure (b), as well as the respective crystallograms after the contour selection (c), (d). 


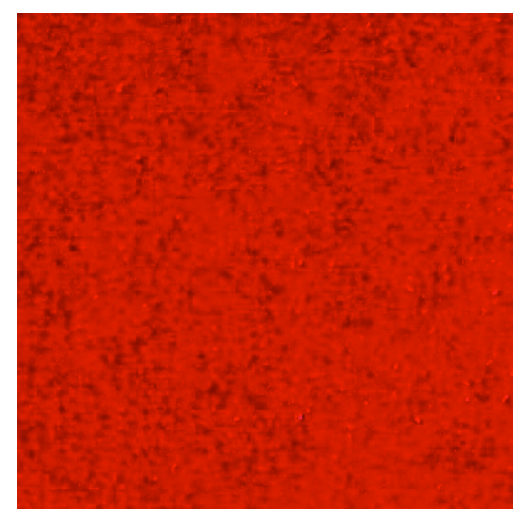

(a)

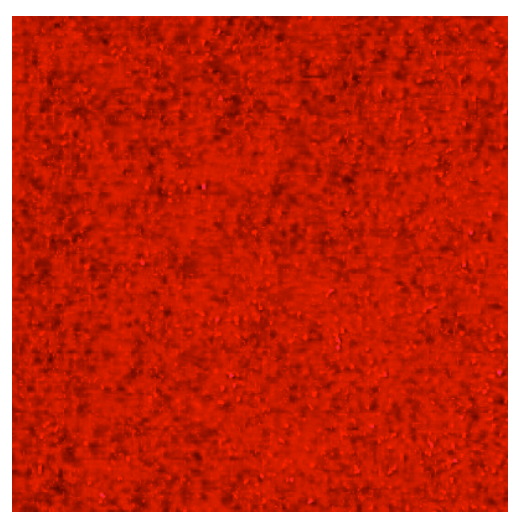

(b)

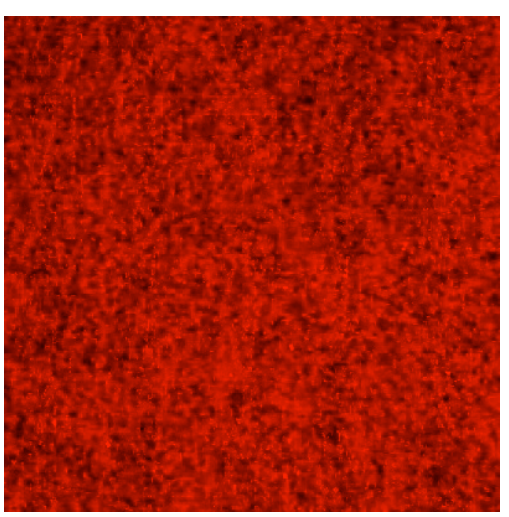

(C)

Fig. 6 Speckle patterns recorded in the course of the gelatine gelling: (a) - immediately after placing the solution into the recording system (gelling just started); (b) - 10 minutes later; (c) - 20 minutes after the beginning of the gelling process.

One can see that at small gelling times (up to 10 minutes) the speckle size decreases, which is due to the unfolding of the gelatine secondary structures. Then the structure evolves into more compact aggregates. Finally, at large times of gelling (more than 20 minutes) the speckle size sharply decreases, which is a fingerprint of the formation of large-scale spatial structures over the entire volume of the solution.

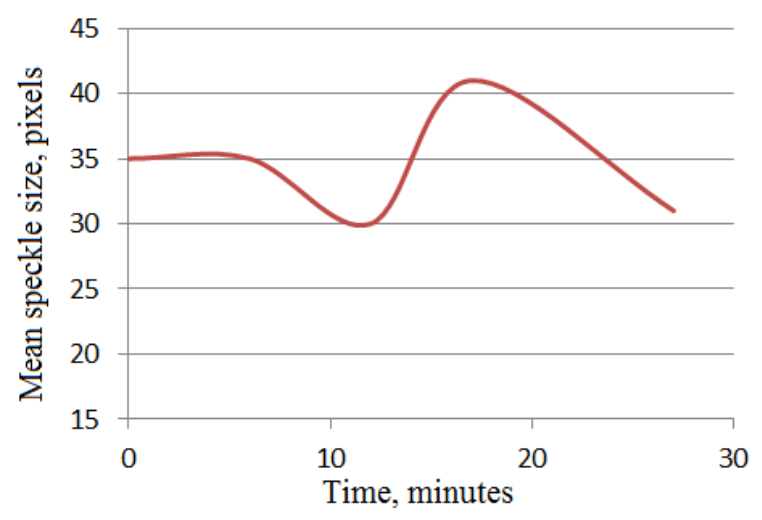

Fig. 6 Dynamics of mean speckle area versus the time of gelatine gelling, calculated using the program OpenCV.

\section{Conclusion}

The proposed method of contour selection and calculating the mean contour size allows the introduction of the quantitative characteristic for comparing the crystallograms from liquids under the different external conditions or at different stages of the study. The algorithm described in the paper can be also applied to develop a numerical criterion for comparing the speckle patterns from different samples of the studied liquid. The advantages of the proposed image processing technique are its high sensitivity, small processing time and clearness of results. 